\title{
Erfahrungen und Konzepte der AOK Hessen
}

Bereits im Jahr 2000 hat die AOK Hessen mit ausgewählten Krankenhäusern Integrierte Versorgungsverträge (IV-Verträge) für elektive HüftTotalendoprothesen (TEPs) geschlossen. Das Ziel dieser IV-Verträge war die Optimierung der Versorgungsqualität für die Patienten. Die Krankenhäuser übernahmen im Rahmen der IV-Verträge u.a. eine Gewährleistungspflicht für das Implantat für die Zeitdauer von 10 Jahren.

\section{Patienten erwarten Qualitätsvergleiche} Im Jahr 2007 hat die AOK Hessen eine repräsentative Befragung ihrer Kunden, die eine Hüft-TEP erhalten hatten, durchgeführt. Im Ergebnis wurde deutlich, dass $84 \%$ der Patienten ein hohes Interesse an Qualitätsinformationen über Krankenhäuser haben und auch anhand der Ergebnisqualität ein Krankenhaus für die eigene Operation aussuchen möchten. Weiterhin wurde deutlich, dass die höchste Kompetenz für Qualitätsvergleiche den Krankenkassen zugeteilt wird (Abb. 1).

Die Qualitätssicherung der stationären Versorgung mit Routinedaten (QSR) bietet für die AOK Hessen die Möglichkeit, diese von den Versicherten geforderten Qualitätsvergleiche transparent darzustellen und anhand weniger, für den „Laien“ verständlichen Ergebnisqualitätsindikatoren zu kommunizieren.

\section{QSR-Berichte als Bestandteil von selektiven Verträgen}

Die Verträge der AOK Hessen zur Integrierten Versorgung nach §140a ff SGB V für die elektiven Hüft-TEPs wurden im Jahr 2008 neu konzipiert. Die bisherige Qualitätsmessung, die für die Krankenhäuser zusätzlichen manuellen Dokumentationsaufwand bedeutete, wurde durch einzelne QSR-Indikatoren (z.B. Wiederaufnahmerate) er- setzt. Die QSR-Indikatoren werden jährlich individuell mit dem Krankenhaus besprochen. Zielsetzung ist, dass die Krankenhäuser im Rahmen der IV-Verträge eine deutlich bessere Ergebnisqualität als der Bundesdurchschnitt erbringen. Die Partnerschaft zwischen den Krankenhäusern und der AOK Hessen ist dadurch gekennzeichnet, dass es zunächst keine vertraglichen Sanktionen bei Nichterreichung der Zielsetzung gibt. Vielmehr sollen die Krankenhäuser die Möglichkeit erhalten, internes Verbesserungspotenzial zu erkennen und zu erschließen.

\section{Pay for Performance}

Perspektivisch wird Pay for Performance (P4P) bei der Preisverhandlung im Rahmen der selektiven Verträge eine Rolle spielen. Die Krankenhäuser werden für überdurchschnittliche Ergebnisqualität entsprechende monetäre Vorteile erhalten. Das heißt, dass mit den Krankenhäusern gestaffelte Preise für eine Hüft-TEP je nach Zielerreichung der QSR-Indikatoren vereinbart werden - je höher die Ergebnisqualität, desto niedriger der Rabatt für die AOK Hessen.

\section{Fazit}

Für die AOK Hessen ist die Messung der Ergebnisqualität im Krankenhaus anhand von stationären Routinedaten das zur Zeit beste in Deutschland vorhandene Instrument. Im Zusammenspiel mit dem P4P-Ansatz werden die Selektiv-Verträge in den nächsten Jahren nicht nur für elektive Leistungen weiter ausgebaut werden.

Autorenerklärung: Der Autor erklärt, dass keine relevanten finanziellen Verbindungen in Bezug auf dieses Manuskript bestehen.

\section{T. Göbel}

Qualitätsmanagement

Schlüsselwörter

Ergebnisqualität

Routinedaten

Selektiv-Verträge

Pay for Performance

Keywords

outcomes of care

administrative data

individual contract

pay for performance

(1)

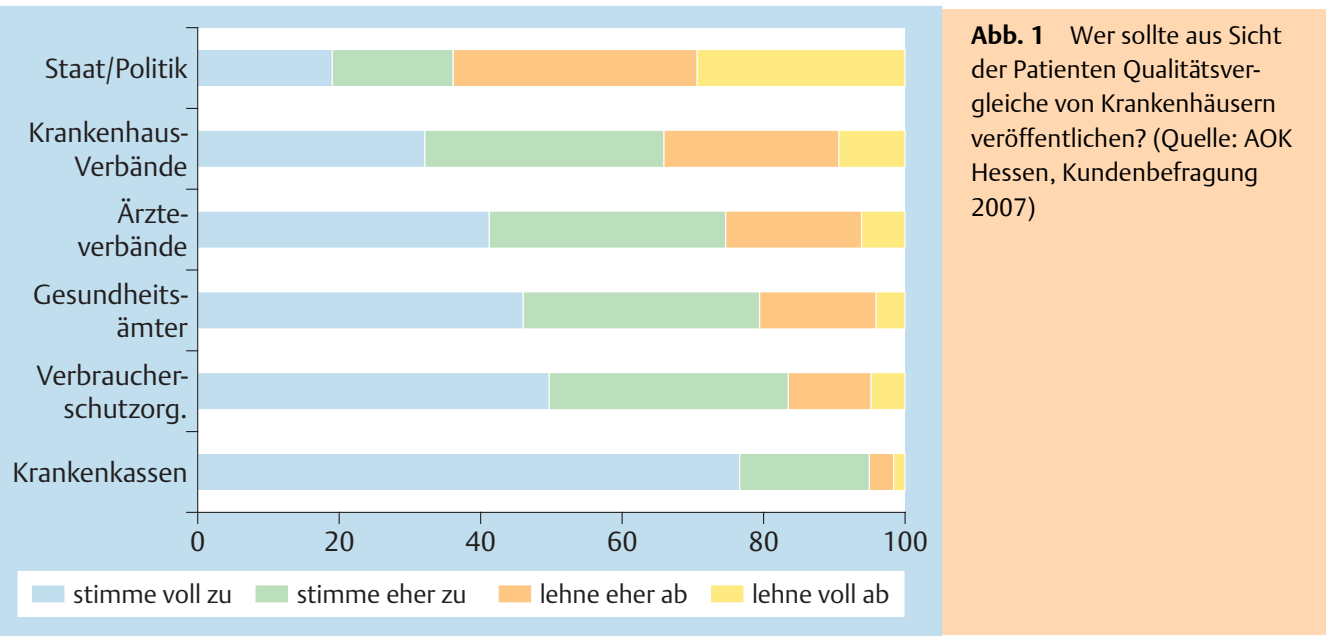

Institut

AOK Hessen, Marburg

Bibliografie

DOI 10.1055/s-0029-1242673 Dtsch Med Wochenschr 2009;

134: S317 - (c) Georg Thieme Verlag KG Stuttgart - New York . ISSN 0012-0472

Korrespondenz Thomas Göbel

AOK - Die Gesundheitskasse in Hessen

Gesundheitspartnermanagement Rollwiesenweg 1 35039 Marburg Tel. 06421/401802 eMail thomas.goebel@ he.aok.de 